Universidade de Brasília

FACE - Faculdade de Administração, Contabilidade e Economia

Departamento de Economia - Programa de Pós-Graduação

\title{
Uma demonstração Alternativa para o Modelo de Contingências Imprecisas
}

Aluno: João Vítor Rego Costa

Orientador: Gil Riella, PhD

Brasília, 2015 


\title{
Universidade de Brasília
}

FACE - Faculdade de Administração, Contabilidade e Economia

Departamento de Economia - Programa de Pós-Graduação

\section{Uma demonstração Alternativa para o Modelo de Contingências Imprecisas}

\author{
Dissertação apresentada ao De- \\ partamento de Economia sob ori- \\ entação do Prof. Dr. Gil Ri- \\ ella como requisito para obtenção \\ do título de Mestre em Ciências \\ Econômicas.
}

Aluno: João Vítor Rego Costa Orientador: Gil Riella, $\mathrm{PhD}$ 
"Uma demonstração Alternativa para o Modelo de Contingências Imprecisas"

JOÃO VÍTOR REGO COSTA

Dissertação apresentada como exigência do curso de Mestrado em Economia da Universidade de Brasília.

Avaliação

BANCA EXAMINADORA

Professor Dr. Gil Riella, UnB

Orientador

Professor Dr. Leandro Gonçalves do Nascimento, UnB

Membro interno

Professor Dr. José Heleno Faro, Insper

Membro externo

Março de 2015

Brasília, DF 


\section{Agradecimentos}

A Vânia e Abiatar, meus pais, pelo apoio absoluto às minhas decisões, mesmo nas contingências mais imprecisas.

Ao Prof. Gil Riella pela generosidade e entusiasmo investidos na pesquisa e formação dos orientandos.

Aos professores José Guilherme de Lara Resende, Leandro Gonçalves do Nascimento e Daniel Oliveira Cajueiro pela dedicação aos alunos, inabalável mesmo nas condições não ideais da vida acadêmica brasileira.

Aos meus companheiros des armes Bruno Furtado e Caio Figueiredo, pela ajuda tão necessária nos últimos dois anos.

À Nayra, pela paciência. 


\section{Resumo}

Redemonstramos o resultado de representação para preferências sobre menus com contingências imprecisas de Epstein et al. (2007) e espaço finito de estados subjetivos. Nossa técnica, que envolve o resultado para preferências incompletas de Kochov (2007) e o axioma Negative Certainty Independence de Dillenberger (2010), permite aplicações do modelo para o caso de preferências variacionais sobre menus, racionalidade subjetiva, e atualização bayesiana de crenças.

Palavras-chave: teoria da decisão, preferências sobre menus, incerteza, ambiguidade, atualização bayesiana.

Classificação JEL: D81 


\begin{abstract}
We provide an alternative demonstration to the representation of preferences over menus with coarse contigencies from Epstein et al. (2007) imposing finitiness to the subjective state space. Our technic - which involves the result for incomplete preferences from Kochov (2007) and the Negative Certainty Independence axiom from Dillenberger (2010) - allows for applications in the case of variational preferences, subjective rationality and bayesian update of priors.
\end{abstract}

Key words: decision theory, preferences over menus, uncertainty, ambiguity, bayesian update.

JEL codes: D81 


\section{Sumário}

1 Introdução $\quad 2$

2 Axiomatização da preferência sobre menus 5

3 Representação funcional de $\succsim \quad \mathbf{9}$

3.1 Representação de preferências incompletas sobre menus . . . . 11

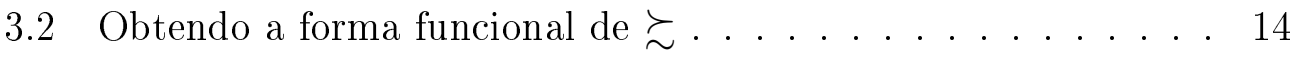

4 Pesquisas futuras e resultados adicionais 18

4.1 Preferências variacionais . . . . . . . . . . . . . 18

4.2 Racionalidade Objetiva e Subjetiva e Preferências sobre Menus 18

4.3 Bayesian Updating . . . . . . . . . . . . . . . 19

5 Observações finais $\quad 22$ 


\section{Introdução}

Como modelar a decisão de um agente cujas alternativas têm resultados que dependem de uma realização futura de estados da natureza e, ao ter que escolher um conjunto dessas alternativas, esse agente o faz sem possuir uma descrição completa desses estados? Para abordar o problema, utilizaremos o framework de preferência sobre menus e alguns dos principais resultados de representação funcional das preferências encontrados na literatura até o momento.

Para compreender a motivação do nosso trabalho, considere o caso de um gerente de investimentos de uma instituição financeira que deve decidir como alocar os recursos de seus clientes. Cada portfólio escolhido, trará retornos condicionados a contingências (políticas, econômicas, institucionais etc) que caracterizarão a economia em um futuro próximo. Contudo, apesar de conseguir conjecturar acerca dos estados da natureza que se realizarão, o gerente não possui uma descrição completa de cada um deles. Há aspectos sutis de cada uma dessas contingências, suficientemente importantes para influenciar o retorno dos portfólios, mas que o agente os desconhece e tem consciência disso. Isto significa que, para cada estado da natureza, não há uma única crença a respeito da probabilidade de sua ocorrência e, consequentemente, dos retornos associados.

Uma primeira abordagem à decisão sobre menus com incerteza foi proposta em Kreps (1979) e Kreps (1992). A sugestão do autor foi axiomatizar a preferência sobre menus de alternativas levando em conta a preferência por flexibilidade, uma hipótese natural a respeito do comportamento de um agente que não tem certeza a respeito dos seus gostos futuros. Na presença de incerteza, os menus, vistos como conjuntos de oportunidades, são tão preferíveis quanto maiores as possibilidades oferecidas por eles. A representação de Kreps (1979), todavia, não capta integralmente nossa motivação, pois, nela, o tomador de decisão age como se houvesse um espaço subjetivo de estados da natureza completamente conhecidos pelo agente, no sentido de não haver ambiguidade dos payoffs associados a eles. Uma abordagem do tipo Savage também seria inadequada pela mesma razão. Ademais, interessa 
ao pesquisador obter um espaço de estados da natureza subjetivo, observável pelo próprio comportamento do agente ex post, quando realizadas as contingências.

De fato, a imprecisão que caracteriza as contingências antecipadas pelo tomador de decisão está associada à ambiguidade presente em modelos de preferência com múltiplas priors, como é o caso de Gilboa e Schmeidler (1989). Veremos que a representação da preferência sobre menus com contingências imprecisas tem um formato semelhante àquele encontrado na modelagem de decisão sobre atos com ambiguidade. Modelaremos nosso problema baseando-nos no trabalho de Epstein et al. (2007) - EMS, daqui por diante - que, por sua vez, generalizaram o arcabouço DLR, de Dekel et al. (2001), no qual os agentes possuem uma preferência sobre menus de loterias derivadas de um espaço de alternativas finito. EMS estendem esse modelo ao incorporar a imprecisão das contingências que se realizarão após a escolha dos menus.

Observe que a utilidade dos menus encontrada em DLR, dada por,

$$
W^{D L R}(x)=\int \max _{\beta \in x} u(\beta) d \mu(u)
$$

toma uma única crença $\mu$ a respeito do conjunto de estados da natureza como suficiente para a tomada de decisão do agente. Isso não é por acaso, pois eles modelam um tomador de decisão que possui uma descrição completa a respeito dos estados, de modo que o retorno de cada loteria para um certo estado é único. EMS incorporam a imprecisão das contingências ao modelar um agente com múltiplas crenças a respeito do retorno das loterias em cada estado e, como em Gilboa e Schmeidler (1989), o agente toma sua decisão com "cautela", visto que a representação de suas preferências sobre menus é do tipo min-max:

$$
W^{E M S}(x)=\min _{\pi \in \Pi} \int \max _{\beta \in x} u(\beta) d \pi(u)
$$

onde $\Pi$ é o conjunto de medidas de probabilidade sobre o espaço subjetivo de estados. 
Um característica comum aos modelos apresentados acima é a de que a preferência sobre menus é completa e, portanto, mesmo no caso de não possuir uma descrição exaustiva das contingências futuras, o agente é capaz de comparar quaisquer dois menus que lhe são oferecidos. Nós construiremos uma demonstração alternativa ao modelo EMS que leva em conta a representação obtida em Kochov (2007) para preferências incompletas sobre menus. À semelhança da decisão sobre atos com múltiplas priors modelada por Gilboa et al. (2010), o trabalho de Kochov (2007) nos fornece uma regra de decisão unânime para os menus. Na sua representação, um menu $x$ é preferível a outro menu $y$ se, e somente se, a utilidade em $x$ é maior ou igual à de $y$ para todas as crenças formadas a respeito do espaço subjetivo de estados. Adicionalmente, faremos a hipótese de que, ao observar um menu de loterias, nosso agente necessita de apenas um número finito delas para avaliar o menu. O axioma de Finitiness nos permitirá concluir que o espaço subjetivo de estados é finito, como veremos adiante.

O restante do trabalho dispõe-se da seguinte forma: na seção 2 descrevemos as primitivas do nosso modelo e, na 3, derivamos o principal resultado a partir da representação de Kochov (2007). Na seção 4, apresentamos três aplicações da técnica envolvida na demonstração e, na seção 5, sugerimos um problema que, se resolvido, poderá estender nosso trabalho. 


\section{Axiomatização da preferência sobre menus}

Modelamos um agente que toma sua decisão em dois estágios: no primeiro, os menus são comparados tendo em vista que, em um segundo momento, após a realização do estado da natureza, uma loteria será escolhida de acordo com a preferência ex post do agente. Seja $B$ um conjunto finito de alternativas e $\Delta(B)$ o conjunto das medidas de probabilidade sobre $B$. $\mathbb{X}$ é a coleção de subconjuntos fechados de $\Delta(B)$, os menus, e $\succsim$ denotará a preferência sobre $\mathbb{X}$. Os axiomas a seguir caracterizam essa relação.

Order $\succsim$ é completa e transitiva.

Continuity Para todo $x,\{y \in \mathbb{X}: y \succsim x\}$ e $\{y \in \mathbb{X}: x \succsim y\}$ são fechados em relação à topologia induzida pela métrica de Hausdorff.

Monotonicity Para quaisquer $x, x^{\prime} \in \mathbb{X} \operatorname{com} x \supseteq x^{\prime}$, temos $x \succsim x^{\prime}$.

Indifference to Randomization (IR) $x \sim c o(x)$, o fecho convexo de $x$.

Nondegeneracy Existem menus $x, x^{\prime} \in \mathbb{X}$ tais que $x \succ x^{\prime}$.

Considere que, para $\lambda \in[0,1]$ e menus $x, y$ quaisquer, o menu $\lambda x+(1-\lambda) y$ refere-se ao conjunto de loterias $\left\{\lambda \beta+(1-\lambda) \beta^{\prime}: \beta \in x\right.$ e $\left.\beta^{\prime} \in y\right\}$.

Preference Convexity $x \succsim x^{\prime} \Rightarrow \lambda x+(1-\lambda) x^{\prime} \succsim x^{\prime}$.

Finiteness Para todo $x$, existe um menu finito $x^{f} \subseteq x$ tal que, para todo $\lambda \in(0,1]$ e qualquer menu $x^{\prime}, \lambda x+(1-\lambda) x^{\prime} \sim \lambda x^{f}+(1-\lambda) x^{\prime}$.

Prevenimos indiferença total ao supor Nondegeneracy. Para um tomador de decisão que não está certo a respeito das probabilidades dos estados futuros, é natural assumir preferência por flexibilidade, como em Kreps (1979). Por conta disso, assumimos monotonicidade da preferência. O axioma de Preference Convexity tem a mesma motivação e traduz a idéia de que o agente tem ganhos de hedging ao misturar dois menus quaisquer, dada a incerteza ex ante que caracteriza o estágio de comparação dos menus. A motivação para Continuity tem caráter técnico. Assumir que a preferência não experimenta 
"saltos"em qualquer par de menus nos permitirá invocar a representação de preferências incompletas de Kochov (2007), como ficará claro adiante.

Ao assumirmos Indifference to Randomization, estamos, implicitamente, fazendo uma hipótese a respeito do timing da incerteza enfrentada pelo agente. Mais especificamente, estamos supondo que quando um estado da natureza se realiza, toda a ambiguidade que marcava a decisão ex ante desaparece, pois, nesse momento, uma descrição completa daquele estado está disponível ao tomador de decisão. A ambiguidade não persiste e, desse modo, o agente antecipa a escolha entre as loterias do menu previamente optado com intuito de maximizar uma utilidade vNM e, portanto, os menus $x$ e $c o(x)$ lhe são indiferentes. Se, contudo, a ambiguidade persiste ex post, IR deixa de ser razoável pois o agente pode experimentar ganhos estritos de randomização no segundo estágio.

Quanto à hipótese de Finitiness, a intuição é a de que, mesmo sem possuir uma descrição completa dos estados subjetivos, nosso agente necessita de apenas um subconjunto finito de loterias dentro de cada menu para avaliálo. Veremos na demonstração do Lema 1 que essa hipótese está diretamente relacionada à estrutura aditiva finita da representação do Teorema 1, visto que ela é condição suficiente para garantir a finitude do espaço de estados subjetivos. Outras formas de Finitiness foram utilizadas na literatura, e.g. Dekel et al. (2009) e Kopylov (2009), onde há uma discussão da relação entre Finiteness e formas aditivas finitas de utilidade.

Adicionalmente, suponha que o tomador de decisão tenha certeza ex ante de que há uma alternativa $b_{*}$ que é o pior resultado ex post - o mesmo vale para a loteria degenerada $\delta_{b_{*}}$. Assumiremos também que o agente saiba $e x$ ante que o menu $\Delta(B)$ lhe trará o melhor resultado ex post, ainda que não conheça qual loteria maximizará sua utilidade após a realização do estado. Consideraremos daqui em diante que $\left\{b_{*}\right\}$ é o menu cujo único elemento é a loteria degenerada $\delta_{b_{*}}$.

Worst Há pelo menos uma alternativa $b_{*} \in B$ tal que $\lambda\left(x \cup\left\{b_{*}\right\}\right)+(1-$ $\lambda) y \sim \lambda x+(1-\lambda) y$ para quaisquer menus $x, y \in \mathbb{X}$ e $\lambda \in(0,1)$. 
Nossa versão de Worst é distinta daquela utilizada por EMS. Aqui, prescindimos da definição de uma relação de dominância sobre as loterias e de posteriormente estendê-la a todo o conjunto de menus, como é feito em Kreps (1979) e em EMS. Além disso, ficará claro na seção seguinte que nossa opção gera uma forma de Worst mais próxima daquela tradicionalmente encontrada na literatura de utilidade esperada, quando começaremos a explorar uma restrição específica de $\succsim$. Quanto à motivação, Worst formaliza a idéia de que o agente não experimenta ganhos de flexibilidade ao incluir em qualquer menu $x$ a loteria degenerada da pior alternativa $b_{*}$. Um raciocínio rápido nos garante que

$$
\Delta(B) \succsim x \succsim\left\{b_{*}\right\} \text { e } \Delta(B) \succ\left\{b_{*}\right\}
$$

para todo $x$. Por Monotonicity, $\Delta(B) \succsim x$. Além disso, dado que o agente está certo de que $b_{*}$ é o pior resultado, $x \succsim\left\{b_{*}\right\}$ vale para todo $x$. Por fim, Monotonicity garante que $\Delta(B) \succsim\left\{b_{*}\right\}$. Caso $\Delta(B) \sim\left\{b_{*}\right\}$, contrariamos Nondegeneracy.

Tendo conhecido o comportamento do agente face aos menus $\Delta(B)$ e $\left\{b_{*}\right\}$, podemos definir o menu certo $x_{p}$ como $x_{p}:=p \Delta(B)+(1-p)\left\{b_{*}\right\}$, i.e. a composição do melhor e pior menu com peso $p \in[0,1]$. Como misturá-los a um menu qualquer não traz ganhos de hedging, assumiremos o seguinte axioma.

Certainty Independence Para $\lambda \in(0,1)$ e $x_{p}=p \Delta(B)+(1-p)\left\{b_{*}\right\}$, temos

$$
x \succsim x^{\prime} \Leftrightarrow \lambda x+(1-\lambda) x_{p} \succsim \lambda x^{\prime}+(1-\lambda) x_{p}
$$

O principal resultado do nosso trabalho é a construção da representação funcional da preferência sobre menus satisfazendo os axiomas acima, baseada em Epstein et al. (2007), conforme o teorema abaixo.

Teorema 1 A preferência $\succsim$ sobre o espaço de menus $\mathbb{X}$ satisfaz Order, Continuity, Monotonicity, Indifference to Randomization, Nondegeneracy, Preference Convexity, Finiteness, Worst $e$ Certainty Independence se, e somente se, existe um conjunto finito de utilidades $N \subseteq\left\{u \in \mathbb{R}_{+}^{B}: u\left(b_{*}\right)=\right.$ 
0 e $\left.\max _{B} u(b)=1\right\}$ e um conjunto fechado e convexo $\Pi$ de medidas de probabilidade sobre $N$ tais que

$$
x \succsim y \Leftrightarrow \min _{\pi \in \Pi} \sum_{u \in N} \pi(u) \max _{\beta \in x} \mathbb{E}_{\beta}(u) \geq \min _{\pi \in \Pi} \sum_{u \in N} \pi(u) \max _{\beta \in y} \mathbb{E}_{\beta}(u)
$$

para quaisquer $x, y \in \mathbb{X}$ e $\mathbb{E}_{\beta}(u)$ a utilidade esperada vNM da loteria $\beta$.

Um resultado importante para a demonstração do Teorema 1 é o de que todo menu $x$ possui um menu certo $x_{p}$ indiferente a ele, o que traduz a idéia de que existe um peso $p$ na mistura entre o pior e melhor resultados suficiente para que o tomador de decisão conjecture receber o mesmo payoff de um menu com menor nível de certeza.

Afirmação 1 Para todo menu $x$, existe $p \in[0,1]$ tal que $x \sim x_{p}=p \Delta(B)+$ $(1-p)\left\{b_{*}\right\}$.

Dem.: Para um menu qualquer $x$, defina $S:=\left\{p \in[0,1]: x_{p} \succsim x\right\}, I:=$ $\left\{p \in[0,1]: x \succsim x_{p}\right\}$ e note que $1 \in S$ e $0 \in I$. Como $\succsim e ́$ continua $e$ completa, podemos afirmar que $S$ e $I$ são fechados e $S \cup I=[0,1]$. Dada a conexidade de $[0,1]$, sabemos que $S \cap I \neq \emptyset$. Portanto, para $p \in S \cap I$, temos que $x \sim x_{p}$.

Na próxima seção, construiremos a representação funcional de $\succsim$ sobre o espaço de menus $\mathbb{X}$ a partir da maior restrição dessa relação invariante com respeito a misturas entre menus, isto é, a maior restrição que satisfaz o axioma da Independência, tradicional na literatura de decisão sob incerteza. 


\section{Representação funcional de $\succsim$}

Suponha que $\succsim$ satisfaz Order, Nondegeneracy, Indifference to randomization, Preference Convexity, Certainty Independence, Continuity, Monotonicity, Worst e Finiteness. Considere agora seu maior subconjunto que satisfaça também o axioma tradicional de independência. Para isso, defina a relação ${ }^{*}$ sobre $\mathbb{X}$ por

$$
x \succsim^{*} x^{\prime} \Leftrightarrow \lambda x+(1-\lambda) y \succsim \lambda x^{\prime}+(1-\lambda) y
$$

para todo $y \in \mathbb{X}$ e $\lambda \in(0,1]$.

Naturalmente, algumas das propriedades de $\succsim$ serão herdadas por sua restrição ${ }^{*}$. Finitiness e Worst, em especial, assumirão formatos mais intuitivos, como veremos em seguida. Contudo, observe que, como a relação primitiva satisfaz independência apenas com relação aos menus certos $x_{p}$, a relação induzida $\succsim^{*}$ não é completa sobre o espaço de menus. Exploramos essas constatações na sequência de afirmações abaixo.

Afirmação 2 ¿*é uma pré-ordem.

Dem.: Pela reflexividade de $\succsim$, é claro que $x \succsim^{*} x$ para todo $x \in \mathbb{X}$. Suponha $x, y$ e $z$ tais que $x \succsim^{*}$ y e $y \succsim^{*} z$. Então, para um menu $x^{\prime}$ qualquer $e$ $\lambda \in(0,1]$, temos $\lambda x+(1-\lambda) x^{\prime} \succsim \lambda y+(1-\lambda) x^{\prime} \succsim \lambda z+(1-\lambda) x^{\prime}$. Para concluir, basta usar a transitividade de $\succsim$.

Afirmação $3 \succsim^{*}$ satisfaz Monotonicity.

Dem.: Isto é consequência imediata da monotonicidade de

Afirmação 4 Sejam $\left\{x^{m}\right\}_{m \in \mathbb{N}} e\left\{y^{m}\right\}_{m \in \mathbb{N}}$ sequências em $\mathbb{X}$ convergentes para $x$ e $y$, respectivamente, tais que $x^{m} \succsim^{*} y^{m} \forall m \in \mathbb{N}$. Então $x \succsim^{*} y$.

Dem.: Pela definição de ${ }^{*}$, temos que para todo $\lambda \in(0,1]$ e qualquer menu $z$, temos

$$
\lambda x^{m}+(1-\lambda) z \succsim \lambda y^{m}+(1-\lambda) z
$$

Como $\succsim$ satisfaz Order e Continuity, concluímos que $\lambda x+(1-\lambda) z \succsim \lambda y+$ 
$(1-\lambda) z$ e, portanto, $x \succsim^{*} y$.

Afirmação $5 \succsim^{*}$ satisfaz Nondegeneracy

Dem.: Pela Afirmação 3, sabemos que $\Delta(B) \succsim^{*}\left\{b_{*}\right\}$. Como $\succsim$ satisfaz Nondegeneracy, temos que $\Delta(B) \succ\left\{b_{*}\right\}$ e, consequentemente, não é verdade que $\left\{b_{*}\right\} \succsim^{*} \Delta(B)$.

Afirmação 6 (Finitiness*) Para todo menu $x$, existe um subconjunto $f$ nito $x^{f}$ tal que $x \sim^{*} x^{\prime}$.

Dem.: Basta utilizar Finitiness de $\succsim$ e a definição de ${ }^{*}$.

Afirmação 7 (Worst*) Para a pior alternativa $b_{*}$, temos $x \cup\left\{b_{*}\right\} \sim^{*} x$.

Dem.: Implicação de Worst em $\succsim$ e da definição de * $^{*}$.

Repare que a Afirmação 3 nos ensina que, se dois menus são $\subseteq$-comparáveis, então também serão $\succsim^{*}$-comparáveis. Além disso, a Afirmação 4 nos mostra que a continuidade de $\succsim$ é preservada em $\succsim^{*}$. Novamente, um raciocínio análogo ao feito para a relação $\succsim$ nos mostra que

$$
\Delta(B) \succsim^{*} x \succsim^{*}\left\{b_{*}\right\} \text { e } \Delta(B) \succ^{*}\left\{b_{*}\right\} .
$$

Vamos, por fim, demonstrar que ${ }^{*}$ satisfaz o axioma da Independência.

Afirmação 8 (Independence) $\quad x \succsim^{*} x^{\prime}$ se, e somente se, $\lambda x+(1-\lambda) y \succsim^{*}$ $\lambda x^{\prime}+(1-\lambda) y$, para quaisquer menus $x, x^{\prime}, y \in \mathbb{X}$ e para todo $\lambda \in[0,1]$.

Dem.: Considere menus $x$ e $x^{\prime}$ tais que $x \succsim^{*} x^{\prime}$. Então, para quaisquer $\lambda, \theta$ $\in(0,1)$ e $y, z \in \mathbb{X}$, temos

$$
\begin{aligned}
\theta(\lambda x+(1-\lambda) y)+(1-\theta) z & =\theta \lambda x+(1-\theta \lambda)\left(\frac{\theta(1-\lambda)}{1-\theta \lambda} y+\frac{1-\theta}{1-\theta \lambda} z\right) \\
& \succsim \theta \lambda x^{\prime}+(1-\theta \lambda)\left(\frac{\theta(1-\lambda)}{1-\theta \lambda} y+\frac{1-\theta}{1-\theta \lambda} z\right) \\
& =\theta\left(\lambda x^{\prime}+(1-\lambda) y\right)+(1-\theta) z .
\end{aligned}
$$


Pela definição de $\succsim^{*}$, concluímos que $\lambda x+(1-\lambda) y \succsim^{*} \lambda x^{\prime}+(1-\lambda) y$. Agora, suponha que $\lambda x+(1-\lambda) y \succsim^{*} \lambda x^{\prime}+(1-\lambda)$ y para $\lambda \in(0,1)$ e um menu y qualquer. Pela Afirmação 5, o conjunto $\left\{\lambda \in[0,1]: \lambda x+(1-\lambda) y \succsim^{*} \lambda x^{\prime}+(1-\lambda) y\right\}$ é um conjunto fechado e, portanto,

$$
\hat{\lambda}:=\max \left\{\lambda \in[0,1]: \lambda x+(1-\lambda) y \succsim^{*} \lambda x^{\prime}+(1-\lambda) y\right\}
$$

está bem definido. Defina ainda $\theta:=\frac{1}{1+\hat{\lambda}}$. Então,

$$
\begin{aligned}
\theta(\hat{\lambda} x+(1-\hat{\lambda}) y)+(1-\theta) x & \succsim^{*} \theta\left(\hat{\lambda} x^{\prime}+(1-\hat{\lambda}) y\right)+(1-\theta) x \\
& =\theta(\hat{\lambda} x+(1-\hat{\lambda}) y)+(1-\theta) x^{\prime} \\
& \succsim^{*} \theta\left(\hat{\lambda} x^{\prime}+(1-\hat{\lambda}) y\right)+(1-\theta) x^{\prime}
\end{aligned}
$$

pela primeira parte desta demonstração. Usando a transitividade de $\succsim^{*} e$ reescrevendo os coeficientes da expressão acima, temos

$$
\frac{2 \hat{\lambda}}{1+\hat{\lambda}} x+\frac{1-\hat{\lambda}}{1+\hat{\lambda}} y \succsim^{*} \frac{2 \hat{\lambda}}{1+\hat{\lambda}} x^{\prime}+\frac{1-\hat{\lambda}}{1+\hat{\lambda}} y
$$

Como $\hat{\lambda}$ é máximo, $\hat{\lambda} \geq \frac{2 \hat{\lambda}}{1+\hat{\lambda}}$ e, consequentemente, $\hat{\lambda}(\hat{\lambda}) \geq 0$. Isto implica que $\hat{\lambda}=1$ e,por $(\star), x \succsim^{*} x^{\prime}$.

\subsection{Representação de preferências incompletas sobre me- nus}

Uma vez exploradas as propriedades de $\succsim^{*}$, podemos enunciar o resultado de Kochov (2007) para representação de preferências incompletas.

Teorema 2 (Kochov (2007)) Uma preordem $\succcurlyeq \subseteq \mathbb{X} \times \mathbb{X}$ satisfaz Continuity, Nondegeneracy, Independence e Monotonicity se, e somente se, existe um conjunto $S$, uma função utilidade dependente de estado $U: \Delta(B) \times S \rightarrow$ $R$ e um conjunto fechado e convexo $\mathcal{M}$ de medidas de probabilidade sobre $S$ tais que 
(i) $x \succcurlyeq y$ se, e somente se,

$$
\int_{S} \max _{\beta \in x} U(\beta, s) d \mu \geq \int_{S} \max _{\beta \in y} U(\beta, s) d \mu \quad \forall \mu \in \mathcal{M}
$$

(ii) cada $U(\cdot, s)$ é uma função utilidade esperada, i.e.

$$
U(\beta, s)=\sum_{b \in B} \beta(b) U(b, s)
$$

Perceba que, do Teorema 2, é possível concluir que o relaxamento da hipótese de completude da preferência tem o mesmo efeito de ambiguidade anteriormente gerado pela imprecisão de contingências, que motiva a representação no Teorema 1. Contudo, apesar de a ambiguidade nos dois casos estar relacionada ao conceito de incerteza Knightiana tratado em Bewley (1986), as representações enunciadas até o momento derivam um espaço de estados subjetivo, observável pelas preferências a posteriori do agente, não sendo necessário tomá-lo como uma primitiva do modelo. Assim como no caso da preferência DLR, o estado subjetivo em Kochov (2007) é único - se duas representações da mesma preferência têm espaços $S$ e $S^{\prime}$ distintos, então eles coincidem no seus fechos, i.e. $\operatorname{cl}(S)=\operatorname{cl}\left(S^{\prime}\right)$. Mais ainda, esse espaço é mínimo no sentido de não haver dois estados $s$ e $s^{\prime}$ que geram uma mesma preferência ex post. No lema abaixo, adaptamos o resultado de Kochov (2007) para a relação ${ }^{*}$.

Lema 1 Existe um conjunto finito de funções $N \subseteq\left\{u \in \mathbb{R}_{+}^{B}: u\left(b_{*}\right)=\right.$ 0 e $\left.\max _{B} u(b)=1\right\}$ e um conjunto fechado e convexo $\Pi$ de medidas de probabilidade sobre $N$ tais que, para todo $x, y \in \mathbb{X}$ :

$$
x \succsim^{*} y \Leftrightarrow \sum_{u \in N} \pi(u) \max _{\beta \in x} \mathbb{E}_{\beta}(u) \geq \sum_{u \in N} \pi(u) \max _{\beta \in y} \mathbb{E}_{\beta}(u) \quad \forall \pi \in \Pi
$$

Dem.: Prosseguiremos a demonstração do lema em três passos.

Passo 1 Vamos mostrar que Finitiness é condição suficiente para que o conjunto $S$ de estados da natureza no Teorema 2 seja finito. Seja $(U, \mathcal{M}, S)$ uma representação de ${ }^{*}$ nos termos do Teorema 2. De Kochov (2007), sa- 
bemos que tal representação pode ser construída sem estados redundantes e apenas com estados relevantes. Isto é, para todo par distinto $s, s^{\prime} \in S, U(\cdot, s)$ e $U\left(\cdot, s^{\prime}\right)$ representam preferências distintas $e$, para todo par de menus $x, y$ com $x \subseteq y$ e $\max _{\beta \in y} U(\beta, s)>\max _{\beta \in x} U(\beta, s)$ para algum $s \in S$, nós temos $y \succ^{*} x$. Considere ainda um menu $x^{*}$ que é uma esfera em $\Delta(B)$, i.e. seja $x^{*}$ tal que exista $\beta^{*} \in \Delta(B)$ e $\delta>0$ com

$$
x^{*}=\left\{\beta \in \Delta(B):\left\|\beta-\beta^{*}\right\| \leq \delta\right\} \subseteq \Delta(B) .
$$

Vamos agora argumentar que o conjunto $S$ necessariamente é finito. Para tanto, note primeiro que, como $x^{*}$ é uma esfera, para cada $s \in S, U(\cdot, s)$ é maximizada por uma única loteria $\beta \in x^{*}$. Similarmente, cada loteria $\beta \in x^{*}$ maximiza, no máximo, uma função em $\{U(\cdot, s): s \in S\}$. Mas então, $x \subseteq x^{*}$ é tal que $x \sim^{*} x^{*}$ somente se

$$
\left\{\beta^{*} \in x^{*}:\left\{\beta^{*}\right\}=\underset{\beta \in x^{*}}{\operatorname{argmax}} U(\beta, s) \text { para algum } s \in S\right\} \subseteq x .
$$

Como, por Finitiness*, existe um menu finito que satisfaz essa condição, concluímos que $S$ é finito. $O$ conjunto $S$ é apenas um conjunto de indice $e$ não tem maior significado, só nos importam as funções utilidade associadas a cada $s \in S$. Portanto, consideraremos no Passo 2 abaixo, que $S$ é simplesmente o conjunto de utilidades sobre loterias geradas a posteriori.

Passo 2 Mas agora note que podemos normalizar os estados da natureza de modo a obter o conjunto $N$ utilizado na representação do Teorema 1. Para isso, escreva:

$$
u(b)=\frac{U(b)-U\left(b_{*}\right)}{\max _{b} U(b)-U\left(b_{*}\right)}
$$

e veja que, de fato, $u\left(b_{*}\right)=0$ e $\max _{B} u(b)=1$. Todavia, isso não necessariamente preserva o ordenamento dos menus e, para corrigir esse problema, teremos de normalizar as medidas de probabilidade em $\mathcal{M}$ da seguinte maneira:

$$
\hat{\pi}(u)=\mu(U)\left[\max _{B} U(b)-U\left(b_{*}\right)\right]
$$

Finalmente, para que as medidas normalizadas somem a unidade, precisamos 
reescrevê-las como abaixo:

$$
\pi(u)=\frac{\hat{\pi}(u)}{\sum_{u \in N} \hat{\pi}(u)} .
$$

$\underline{\text { Passo } 3}$ Dos Passos 1 e 2, podemos reescrever o resultado do Teorema 2 da seguinte maneira

$$
x \succsim^{*} y \text { se, e somente se, } \sum_{u \in N} \mu(u) \max _{\beta \in x} \mathbb{E}_{\beta}(u) \geq \sum_{u \in N} \mu(u) \max _{\beta \in y} \mathbb{E}_{\beta}(u)
$$

mantendo o formato de utilidade esperada para $u \in N$.

\subsection{Obtendo a forma funcional de $\succsim$}

Seja $w: \mathbb{X} \times \Pi \rightarrow \mathbb{R}$ a função definida por

$$
w(x, \pi)=\sum_{u \in N} \pi(u) \max _{\beta \in x} \mathbb{E}_{\beta}(u)
$$

Vamos examinar o valor que ela assume nos menus certos $x_{p}$, para cada $\pi \in \Pi:$

$$
\begin{aligned}
w\left(x_{p}, \pi\right) & =\sum_{u \in N} \pi(u) \max _{\beta \in x_{p}} \mathbb{E}_{\beta}(u) \\
& =\sum_{u \in N} \pi(u) \max _{\beta \in x_{p}} \mathbb{E}_{p \beta^{\prime}+(1-p) \delta_{b_{*}}}(u), \quad \beta^{\prime} \in \Delta(B) \\
& =\sum_{u \in N} \pi(u) \max _{\beta \in x_{p}}\left\{p \sum_{b \in B} \beta^{\prime}(b) u(b)+(1-p) \sum_{b \in B} \delta_{b_{*}}(b) u(b)\right\} \\
& =\sum_{u \in N} \pi(u) p \max _{\beta^{\prime} \in \Delta(B)} \sum_{b \in B} \beta^{\prime}(b) u(b), \quad \text { pois } u\left(b_{*}\right)=0 \text { e } \delta_{b_{*}}(b)=0 \forall b \neq b_{*} \\
& =\sum_{u \in N} \pi(u) p \max _{\beta^{\prime} \in \Delta(B)} \mathbb{E}_{\beta^{\prime}}(u) \\
& =p \sum_{u \in N} \pi(u) \cdot 1 \\
& =p
\end{aligned}
$$


donde a penúltima igualdade é consequência do fato de que o elemento que maximiza $u\left(\beta^{\prime}\right)$ é a loteria degenerada $\delta_{\bar{b}}$ na qual $\bar{b} \in \operatorname{argmax} u(b)$, ou seja, $u(\bar{b})=1$. É importante notar ainda que $w\left(x_{p}, \pi\right)=p$ para qualquer prior $\pi \in \Pi$. Portanto, podemos afirmar que para dois menus certos $x_{p}$ e $x_{p^{\prime}}$, temos que $x_{p} \succsim^{*} x_{p^{\prime}}$ se, e somente se, $p \geq p^{\prime}$.

Tendo estudado o valor que $w$ assume sobre os menus certos $x_{p}$, precisamos ainda de uma propriedade da relação primitiva $\succsim$ referente a misturas convexas entre menus. Mais precisamente, mostramos abaixo que o Lema 1, Preference Convexity e Certainty Independence são suficientes para afirmar que se um menu é $\succsim$-preferido a um menu certo, misturá-los a um terceiro menu com pesos iguais mantém a relação invariante. Mais precisamente, mostramos que as primitivas de nosso modelo implicam no axioma Negative Certainty Independence de Dillenberger (2010).

Lema 2 A relação $\succsim$ satisfaz Negative Certainty Independence (NCI), i.e. se $x \succsim x_{p}$, então $\lambda x+(1-\lambda) y \succsim \lambda x_{p}+(1-\lambda) y$ para todo $\lambda \in(0,1)$ e $y \in \mathbb{X}$.

Dem.: Tome $x$ e $x_{p}$ em $\mathbb{X}$, com um $p$ qualquer no intervalo $[0,1]$, tais que $x \succsim x_{p}$. Pela Afirmação 1 , sabemos que existe $\bar{p} \in[0,1]$ tal que $x \sim x_{\bar{p}}$. Logo, $x \sim x_{\bar{p}} \succsim x_{p}$. Como $\succsim$ satisfaz Certainty Independence, isto implica que $\bar{p} \geq p$. Da observação que fizemos antes do lema, sabemos que, por sua vez, isto implica que $x_{\bar{p}} \succsim^{*} x_{p}$. Se $y \sim x \sim x_{\bar{p}}$, então $x_{\bar{p}}=\lambda x_{\bar{p}}+(1-$ ג) $x_{\bar{p}} \sim \lambda x_{\bar{p}}+(1-\lambda) y$, por Certainty Independence. Preference Convexity nos permite afirmar que $\lambda x+(1-\lambda) y \succsim x \sim x_{\bar{p}} \sim \lambda x_{\bar{p}}+(1-\lambda) y$. Usando transitividade e a discussão no parágrafo anterior, chegamos em $\lambda x+(1-$ $\lambda) y \succsim \lambda x_{p}+(1-\lambda) y$. Contudo, se não vale que $y \sim x$, então considere $o$ ato simples $x_{p^{\prime}}:=\left(\frac{\theta}{1-\theta}\right) x_{\hat{p}}+\left(\frac{1-2 \theta}{1-\theta}\right) x_{\bar{p}}$, com $\theta \in\left(0, \frac{1}{2}\right)$ e $x_{\hat{p}}$ o menu simples 
tal que $y \sim x_{\hat{p}}$. Observe que

$$
\begin{aligned}
\theta x_{\bar{p}}+(1-\theta) x_{p^{\prime}} & =\theta x_{\bar{p}}+(1-\theta)\left[\left(\frac{\theta}{1-\theta}\right) x_{\hat{p}}+\left(\frac{1-2 \theta}{1-\theta}\right) x_{\bar{p}}\right] \\
& =\theta x_{\bar{p}}+\theta x_{\hat{p}}+(1-2 \theta) x_{\bar{p}} \\
& =\theta x_{\hat{p}}+(1-\theta) x_{\bar{p}} \\
& \sim \theta y+(1-\theta) x_{\bar{p}}, \text { por Certainty Independence. }
\end{aligned}
$$

Aplicando Certainty Independence mais uma vez, temos

$$
\theta x+(1-\theta) x_{p^{\prime}} \sim \theta x_{\bar{p}}+(1-\theta) x_{p^{\prime}} \sim \theta y+(1-\theta) x_{\bar{p}}
$$

Ao aplicarmos Preference Convexity na expressão acima, obtemos

$$
\begin{aligned}
\lambda\left(\theta x+(1-\theta) x_{p^{\prime}}\right) & +(1-\lambda)\left(\theta y+(1-\theta) x_{\bar{p}}\right) \succsim \theta x_{\bar{p}}+(1-\theta) x_{p^{\prime}} \\
& =\lambda\left(\theta x_{\bar{p}}+(1-\theta) x_{p^{\prime}}\right)+(1-\lambda)\left(\theta x_{\bar{p}}+(1-\theta) x_{p^{\prime}}\right) \\
& \sim \lambda\left(\theta x_{\bar{p}}+(1-\theta) x_{p^{\prime}}\right)+(1-\lambda)\left(\theta y+(1-\theta) x_{\bar{p}}\right)
\end{aligned}
$$

cuja última linha é consequência de Certainty Independence. Podemos reescrever a expressão acima da seguinte forma

$\theta(\lambda x+(1-\lambda) y)+(1-\theta)\left(\lambda x_{p^{\prime}}+(1-\lambda) x_{\bar{p}}\right) \succsim \theta\left(\lambda x_{\bar{p}}+(1-\lambda) y\right)+(1-\theta)\left(\lambda x_{p^{\prime}}+(1-\lambda) x_{\bar{p}}\right)$

donde Certainty Independence nos permite afirmar que $\lambda x+(1-\lambda) y \succsim$ $\lambda x_{\bar{p}}+(1-\lambda) y$. Rocorde-se que $\lambda x_{\bar{p}}+(1-\lambda) y \succsim \lambda x_{p}+(1-\lambda) y$, do início

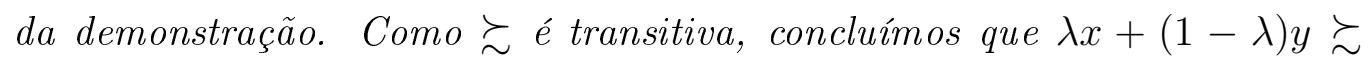
$\lambda x_{p}+(1-\lambda) y$ para todo $\lambda \in(0,1)$ e $y \in \mathbb{X}$.

Munidos dos Lemas 1 e 2, podemos, por fim, estabelecer a forma funcional descrita no Teorema 1 para a representação de $\succsim$.

Demonstração do Teorema 1: Vamos agora estabelecer a representação da relação $\succsim$ original a partir dos resultados do Lema 1 e do Lema 2. Recorde que, de $N C I$, aprendemos que as relações $\succsim \mathrm{e} \succsim^{*}$ coincidem para os menus 
certos, ou seja

$$
\begin{aligned}
x_{p} \succsim x_{\bar{p}} & \Leftrightarrow p \geq \bar{p} \\
& \Leftrightarrow w\left(x_{p}, \pi\right) \geq w\left(x_{\bar{p}}, \pi\right) \forall \pi \in \Pi \\
& \Leftrightarrow x_{p} \succsim^{*} x_{\bar{p}}
\end{aligned}
$$

Agora, fixe $x \in \mathbb{X}$ e $p \in[0,1]$ tal que $x \sim x_{p}$. Sabemos que $x \succsim^{*} x_{p}$. Logo,

$$
w(x, \pi) \geq w\left(x_{p}, \pi\right)=p \quad \forall \pi \in \Pi
$$

e, consequentemente, $\min _{\pi \in \Pi} w(x, \pi) \geq p$. Mas, agora, suponha que

$$
\min _{\pi \in \Pi} w(x, \pi)>p
$$

Então, para qualquer $p^{\prime} \in\left(p, \min _{\pi \in \Pi} w(x, \pi)\right)$, temos que $x_{p^{\prime}} \succ^{*} x_{p} \sim^{*} x$, uma contradição. Portanto,

$$
\min _{\pi \in \Pi} \sum_{u \in N} \pi(u) \max _{\beta \in x} u(\beta)=p
$$

Argumentos usuais da literatura de menus garantem que a forma funcional acima implica na axiomatização de $\succsim$. 


\section{Pesquisas futuras e resultados adicionais}

\subsection{Preferências variacionais}

A técnica de demonstração que utilizamos na presente dissertação indica um caminho pelo qual é possível obter uma versão do modelo de preferências variacionais de MMR - Maccheroni et al. (2006) - no espaço de preferências sobre menus. Os passos seriam os seguintes: utilizar a axiomatização das preferências variacionais Bewley de Faro (2015) no mundo de menus para, em seguida, adaptar a demonstração de Brotherhood (2014) e obter uma versão das preferências variacionais de MMR.

\subsection{Racionalidade Objetiva e Subjetiva e Preferências sobre Menus}

Outro exercício que podemos fazer utilizando as técnicas dessa dissertação consiste em obter uma versão do principal resultado de Gilboa et al. (2010) no mundo de menus. Para tanto, considere um par de preferências $\succsim, \succsim^{*} \subseteq \mathbb{X} \times \mathbb{X}$. nós vamos assumir que ${ }^{*}$ satisfaz os axiomas da seção 3 (afirmações 2 a 8 ) e que $\succsim$ satisfaz Order e Continuity. Adicionalmente, assumiremos as seguintes propriedades que relacionam as duas preferências:

Consistency Para todo par de menus $x, y \in \mathbb{X}, x \succsim^{*} y$ implica que $x \succsim y$.

Default to Certainty Para todo menu $x$ e menu certo $x_{p}$, se não é verdade que $x \succsim^{*} x_{p}$, então $x_{p} \succ x$.

Nós, agora, podemos enunciar o seguinte resultado:

Teorema 3 As seguintes afirmações são equivalentes:

(i) As relações satisfazem os postulados acima e, juntas, satisfazem Consistency e Default to Certainty;

(ii) Existe um conjunto finito de funções $N \subseteq\left\{u \in \mathbb{R}_{+}^{B}: u\left(b_{*}\right)=0\right.$ e $\max _{B} u(b)=$ $1\}$ e um conjunto fechado e convexo $\Pi$ de medidas de probabilidade sobre 
$N$ tais que, para todo $x, y \in \mathbb{X}$ :

$$
x \succsim^{*} y \Leftrightarrow \sum_{u \in N} \pi(u) \max _{\beta \in x} \mathbb{E}_{\beta}(u) \geq \sum_{u \in N} \pi(u) \max _{\beta \in y} \mathbb{E}_{\beta}(u) \quad \forall \pi \in \Pi .
$$

e

$$
x \succsim y \Leftrightarrow \min _{\pi \in \Pi} \sum_{u \in N} \pi(u) \max _{\beta \in x} \mathbb{E}_{\beta}(u) \geq \min _{\pi \in \Pi} \sum_{u \in N} \pi(u) \max _{\beta \in y} \mathbb{E}_{\beta}(u)
$$

A demonstração do teorema acima segue exatamente os passos em Gilboa et al. (2010) e, por isso, será omitida aqui.

\subsection{Bayesian Updating}

Há ainda uma terceira aplicação da nossa técnica que consiste em obter o resultado de atualização bayesiana de priors - Riella (2013) - para o caso de contingências imprecisas. Com esse intuito, defina uma preferência $\succcurlyeq$ que admite representação finita de utilidade esperada aditiva (PAEU) da seguinte maneira:

Definição Uma relação $\succcurlyeq \subseteq \mathbb{X} \times \mathbb{X}$ é PAEU se existe um conjunto $N \subseteq$ $\left\{u \in \mathbb{R}_{+}^{B}: u\left(b_{*}\right)=0\right.$ e $\left.\max _{B} u(b)=1\right\}$ e um conjunto fechado e convexo $\Pi$ de medidas de probabilidade sobre $N$ tais que:

1. $x \succcurlyeq y \Leftrightarrow$

$$
\min _{\pi \in \Pi} \sum_{u \in N} \pi(u) \max _{\beta \in x} \mathbb{E}_{\beta}(u) \geq \min _{\pi \in \Pi} \sum_{u \in N} \pi(u) \max _{\beta \in y} \mathbb{E}_{\beta}(u)
$$

2. $\bigcup_{\pi \in \Pi} \operatorname{supp}(\pi)=N$ e, para $u$ e $u^{\prime}$ distintas, u não é uma transformação positiva afim de $u^{\prime}$.

Uma preferência PAEU sobre menus com contingências imprecisas é, portanto, uma preferência que satisfaz os axiomas do nosso modelo e, para cada estado no espaço subjetivo, existe pelo menos uma prior que o tenha em seu suporte. Para ver que, de fato, a condição 2 acima é atendida pela preferência do Teorema 1, suponha que exista um estado $\bar{u} \in N$ tal que $\pi(\bar{u})=0$ 
para toda $\pi \in \Pi$. Agora redefina as priors em $\Pi$ da seguinte maneira:

$$
\tilde{\pi}(u)=\pi(u) \forall u \in N \backslash\{\bar{u}\}
$$

Mas então repare que basta-nos o espaço de estados $N \backslash\{\bar{u}\}$ e o conjunto de medidas de probabilidade $\tilde{\pi}$, digamos $\tilde{\Pi}$, para satisfazer a representação do Teorema 1. Entretanto, isto contradiz a unicidade de $N$ decorrente de Kochov (2007).

Sejam, então, duas relações PAEU $\succcurlyeq e t^{*}$, com a última sendo a preferência sobre menus após o agente receber um sinal objetivo a respeito das contingências futuras. Como na demonstração do Teorema 1, vamos identificar em cada uma delas suas maiores restrições que satisfazem independência, denotadas por $\succcurlyeq_{r} \mathrm{e} \succcurlyeq_{r}^{*}$, respectivamente. Como nossa definição de PAEU admite invariância da relação para misturas com menus certos apenas, as restrições $\succcurlyeq_{r} \mathrm{e} \succcurlyeq_{r}^{*}$ são incompletas. O axioma abaixo as relaciona.

Flexibility Consistency (Moura e Riella, 2013) Para quaisquer menus $x, y \in \mathbb{X}, x \succcurlyeq_{r}^{*} y$ e não $x \succcurlyeq_{r} y$ ou $y \succcurlyeq_{r}^{*} x$ e não $y \succcurlyeq_{r} x$ implicam que existe um menu $z$ tal que $x \cup y \cup z \sim_{r}^{*} x \cup z$, mas $x \cup y \cup z \succ_{r} x \cup z$.

Flexibility Consistency impõe que para quaisquer discordâncias entre as preferências $\succcurlyeq_{r} \mathrm{e} \succcurlyeq_{r}^{*}$, elas decorrem do fato de que a restrição posterior ao recebimento do sinal enxerga ganhos menores de flexibilidade, valoriza "menos" a flexibilidade. Finalmente, podemos utilizar o seguinte resultado.

Teorema 4 (Moura e Riella (2013)) As afirmações abaixo são equivalentes:

1. Sejam $N$ e $N^{*}$ os espaços de estados subjetivos de $\succcurlyeq_{r} e \succcurlyeq_{r}^{*}$, respectivamente. Para quaisquer menus $x$ e $y$ com

$$
\max _{\beta \in x} \mathbb{E}_{\beta}(u)=\max _{\beta \in y} \mathbb{E}_{\beta}(u) \forall u \in N \backslash N^{*},
$$

$x \succcurlyeq_{r} y \Leftrightarrow y \succcurlyeq_{r}^{*} x$ 
2. Para toda representação $(N, \Pi)$ de $\succcurlyeq_{r}$, existe $M \subseteq N$ tal que $\left(M, \Pi_{M}\right)$ representa $\succcurlyeq_{r}^{*}$, onde $\Pi_{M}$ é o conjunto de priors $\pi \in \Pi \operatorname{com} \pi(M)>0$ atualizadas pela regra de Bayes.

Concluímos que, portanto, na presença de contingências imprecisas, o sinal objetivo gera uma preferência cujo espaço de estados subjetivo é um subconjunto daquele anteriormente gerado na ausência do sinal. Mais ainda, o conjunto de priors definidas sobre o novo espaço de estados é simplesmente a atualização bayesiana das priors definidas sobre o antigo espaço. 


\section{Observações finais}

A representação obtida no Teorema 1 acrescenta à literatura de utilidades aditivas finitas o tratamento de preferências sobre menus com contingências imprecisas. Apesar de semelhante àquela encontrada em EMS, nossa representação exige mais estrutura da relação de preferência, visto que assumimos o axoma de Finitiness. Todavia, apesar de utilizarmos uma versão mais forte de continuidade - EMS assumem que os contornos superior e inferior são fechados pelo menos para os menus certos, e não para qualquer menu, como é o nosso caso - é um engano acreditar que isso acarreta em mais estrutura para a preferência. De fato, a versão fraca de continuidade em EMS em conjunto com o axioma de Certainty Independence são suficientes para fazer valer a forma mais forte que assumimos ${ }^{1}$. A opção de axiomatização que fizemos, contudo, nos permitiu aproveitar o resultado da representação para preferências incompletas de Kochov (2007), à semelhança do que é feito no caso de preferências sobre atos, onde a representação em Bewley (1986) pode ser usada como degrau para se chegar ao resultado clássico de Gilboa e Schmeidler (1989) ${ }^{2}$.

Recorde-se que, ao assumir IR na preferência, estamos supondo que a ambiguidade não persiste após a realização de um estado no espaço subjetivo do agente. Se, entretanto, a imprecisão das contingências remanesce no estágio de escolha das loterias, EMS mostram que é possível obter a seguinte representação

$$
W(x)=\int \min _{\pi \in \Pi} \max _{\beta \in x} u(\beta) d \pi(u)
$$

abrindo-se mão de $I R$. Um exame de como Finitiness e o resultado de Kochov (2007) podem ser utilizados no caso de imprecisão persistente das contingências ainda está por ser feito.

\footnotetext{
${ }^{1}$ Para ver isto, basta refazer os passos iniciais da demonstração do Teorema da Utilidade Esperado, utilizando os menus $\Delta(B)$ e $\left\{b_{*}\right\}$ como melhor e pior menus, respectivamente. Adicionalmente, repare que podemos demonstrar a unicidade do menu indiferente $x_{p}$ na Afirmação 1.

${ }^{2}$ Para uma versão completa dessa demonstração, ver Riella (2014).
} 


\section{Referências}

Bewley, T. F. (1986). Knightian Decision Theory: Part I.

Brotherhood, L. M. M. (2014). Uma Demonstração Alternativa para a Representação de Preferências Variacionais. Ph. D. thesis, University of Brasília.

Dekel, E., B. Lipman, and A. Rustichini (2001). Representing preferences with a unique subjective state space. Econometrica 69(4), 891-934.

Dekel, E., B. L. Lipman, and A. Rustichini (2009). Temptation-driven preferences. Review of Economic Studies 76, 937-971.

Dillenberger, D. (2010). Preferences for One-Shot Resolution of Uncertainty and Allais-Type Behavior. Econometrica 78 (6), 1973-2004.

Epstein, L. G., M. Marinacci, and K. Seo (2007). Coarse contingencies and ambiguity. Theoretical Economics 2, 355-394.

Faro, J. H. (2015). Variational Bewley Preferences. Journal of Economic Theory 157, 699-729.

Gilboa, I., F. Maccheroni, M. Marinacci, and D. Schmeidler (2010). Objective and Subjective Rationality in a Multiple Prior Model. Econometrica 78(2), $755-770$.

Gilboa, I. and D. Schmeidler (1989). Maxmin expected utility with nonunique prior. Journal of mathematical economics (December).

Kochov, A. S. (2007). Subjective States without the Completeness Axiom.

Kopylov, I. (2009). Finite additive utility representations for preferences over menus. Journal of Economic Theory 144(1), 354-374.

Kreps, D. M. (1979). A Representation Theorem for 'Preference for Flexibility'. Econometrica 47(3), 565-577. 
Kreps, D. M. (1992). Static choice in the presence of unforeseen contingencies. In Economic Analysis of Markets and Games: Essays in Honor of Frank Hahn, pp. 258-281. MIT Press.

Maccheroni, F., M. Marinacci, and A. Rustichini (2006). Ambiguity aversion, robustness, and the variational representation of preferences. Econometrica 74 (6), 1447-1498.

Moura, F. S. D. and G. Riella (2013). Preference for Flexibility and Dynamic Consistency with Incomplete Preferences.

Riella, G. (2013). Preference for Flexibility and Dynamic Consistency. Journal of Economic Theory 148(6), 2467-2482.

Riella, G. (2014). Notas de Aula do Curso de Teoria da Decisão - UnB. 